ESAIM: PROCEEDINGS AND SURVEYS, September 2014, Vol. 45, p. 300-307

J.-S. Dhersin, Editor

\title{
SHAPE DEFORMATION AND OPTIMAL CONTROL
}

\author{
Sylvain Arguillère ${ }^{1}$, Emmanuel Trélat ${ }^{2}$, Alain Trouvé ${ }^{3}$ and Laurent Younès ${ }^{4}$
}

\begin{abstract}
Shape deformation analysis is concerned with determining a deformation of a given shape into another one, which is optimal for a certain cost. We provide the main ideas for a new general approach to shape deformation analysis, using the framework of optimal control theory.

This point of view can be made independent from the parametrization of the shape, and allows to model general constrained shape analysis problems. The use of a infinite dimensional variant of the constrained Pontryagin Maximum Principle characterizes the optimal solutions of the shape deformation problem in a very general way.

Résumé. L'analyse des déformations consiste à déterminer une transformation d'une forme donnée en une autre, optimale pour un certain coût. Nous donnons ici les idées principales pour une nouvelle approche générale pour cette analyse à l'aide de la théorie du contrôle optimal.

Ce point de vue peut être pris de manière à ne pas dépendre de la paramétrisation de la forme, et permet également de modéliser des contraintes dans les problèmes d'analyse de déformations. Nous utilisons une variante en dimension infinie (cadre naturel de l'analyse des déformations) du principe du maximum de Pontryagin avec contraintes permettant de caractériser de façon très générale les déformations optimales recherchées.
\end{abstract}

\section{INTRODUCTION}

The mathematical analysis of shapes has become a subject of growing interest in the past few decades, and has motivated the development of efficient image acquisition and segmentation methods, with applications to many domains, including computational anatomy and object recognition.

The general purpose of shape analysis is to compare two (or more) shapes embedded in a space $\mathbb{R}^{d}$ in a way that takes into account their geometric properties, or how "similar" they look. In Shape deformation analysis, a cost for every possible deformation of a shape is considered, and the problem is to find the deformation which minimizes this cost.

Taking a hint from fluid dynamics, we will consider deformations given by flows of diffeomorphisms generated by time-dependent vector fields (see $[6,15,16]$ ): deformations of the whole space, like diffeomorphisms, induce deformations of the shape itself.

\footnotetext{
${ }^{1}$ Université Pierre et Marie Curie (Univ. Paris 6), CNRS UMR 7598, Laboratoire Jacques-Louis Lions, F-75005, Paris, France (sylvain.arguillere@upmc.fr).

${ }^{2}$ Université Pierre et Marie Curie (Univ. Paris 6) and Institut Universitaire de France and Team GECO Inria Saclay, CNRS UMR 7598, Laboratoire Jacques-Louis Lions, F-75005, Paris, France (emmanuel.trelat@upmc.fr).

${ }^{3}$ Ecole Normale Supérieure de Cachan, Centre de Mathématiques et Leurs Applications, CMLA, 61 av. du Pdt Wilson, F-94235 Cachan Cedex, France (trouve@cmla.ens-cachan.fr).

${ }^{4}$ Johns Hopkins University, Center for Imaging Science, Department of Applied Mathematics and Statistics, Clark 324C, 3400 N. Charles st. Baltimore, MD 21218, USA (laurent.younes@jhu.edu).
}

(C) EDP Sciences, SMAI 2014 
This framework has led to the development of a family of registration algorithms called Large Deformation Diffeomorphic Metric Mapping (LDDMM), in which the correspondence between two shapes comes from the minimization of an objective functional that is the sum of two terms (see $[4,5,9,11,12]$ ). The first term is the total kinetic energy of the deformation (the $L^{2}$-norm of the vector field in the case of fluid dynamics, though ours will usually be different). The second term is a data attachment penalizing the difference between the deformed shape and a target.

This framework allows one to use tools from Riemannian geometry (see [17]), along with classical results from the theory of Lie groups equipped with right-invariant metrics (see $[2,3,8,10,18]$ ). However, our approach uses control theory, in particular optimal control (see preliminary ideas in [17]).

While accounting for some of the geometric information in the shape, like singularities, it does not consider other intrinsic properties of the studied shape, which can also depend on the nature of the object modeled by the shape: one may wish the total volume of the shape to remain constant (water balloon), or the movement to occur only along certain directions (fibers in a muscle). It is therefore important to be able to add constraints to the problem.

The purpose of this paper is to summarize and report on the authors's results in [1]. Since shape analysis has a natural setting in infinite dimension (indeed, the shape space is typically the space of all submanifolds in $\mathbb{R}^{d}$ ), we will need to derive an appropriate infinite dimensional variant of the Pontryagin Maximum Principle (or PMP) with an infinite number of equality constraints.

\section{The POINT OF VIEW OF OPTIMAL CONTROL FOR SHAPE DEFORMATION}

In this paper, a shape space $M$ is a space of continuous maps from a smooth compact Riemannian manifold $S$ into an Eucliddean space $\mathbb{R}^{d}$. The elements of $M$ are states of the shape, denoted by $q: S \rightarrow \mathbb{R}^{d}$. For example, if $S$ is the unit circle then $M$ is the set of all parametrized continuous closed curves in $\mathbb{R}^{d}$. When $S$ has dimension 0 , it is a finite union of points $\left\{s_{1}, \ldots, s_{n}\right\}$. In this case, $M=\left(\mathbb{R}^{d}\right)^{n}$ is finite dimensional. This is the most important case for practical applications, and it is frequently used to approximate infinite dimensional problems.

Deformation through the group action of diffeomorphisms. The group of diffeomorphisms has a left action on the shape space by composition: for $\varphi \in \operatorname{Diff}\left(\mathbb{R}^{d}\right)$ and $q \in M=\mathcal{C}^{0}\left(S, \mathbb{R}^{d}\right)$, we have $\varphi \cdot q=\varphi \circ q$. The map $q \mapsto \varphi \cdot q$ is of class $\mathcal{C}^{\ell}$ whenever $\varphi$ is of class $\mathcal{C}^{\ell}$.

A deformation of $\mathbb{R}^{d}$ is a one-parameter family of diffeomorphisms $(\varphi(t))_{t \in[0,1]}$ with $\varphi(0)=I d_{\mathbb{R}^{d}}$. Such a deformation produces a deformation $q:[0,1] \rightarrow M$ of an initial shape $q_{0}$ by letting $q(t)=\varphi(t) \circ q_{0}$.

A classical problem of shape deformation is to find an "optimal" deformation of an initial state $q_{0}$ into a final state close to a target $q_{1}$. More precisely, we will want to minimize a function of the form $E(\varphi)+g(q(1))$, over all deformations $\varphi=(\varphi(t))_{t \in[0,1]}$ of $\mathbb{R}^{d}$. Here, $E$ is a functionnal representing the kinetic energy of the deformation, $q(1)=\varphi(1) \circ q_{0}$ is the final state, and $g$ is a data attachment measuring how close the final state is from the target $q_{1}$. To define $E$, we take a hint from fluid mechanics and use Euler-Lagrange coordinates.

Induced control system. Let $v$ be a bounded vector field of class $\mathcal{C}^{1}$. The infinitesimal action of $v$ on a state $q \in M$, denoted $\xi_{q} v$, is defined as the composition $\xi_{q} v=v \circ q$. It is also the velocity vector at 0 of the curve $t \mapsto \varphi_{v}(t) \cdot q$, where $\varphi_{v}(t, \cdot)$ is the flow of $v$.

Example 1. An interesting case is when $S=\left(s_{1}, \ldots, s_{n}\right)$ is a zero-dimensional manifold and

$$
M=\operatorname{Lmk}_{d}(n)=\left(\mathbb{R}^{d}\right)^{n}
$$

is the (so-called) space of $n$ landmarks in $\mathbb{R}^{d}$. For $q=\left(x_{1}, \ldots, x_{n}\right)$, the action of a diffeomorphism $\varphi$ is given by $\varphi \cdot q=\left(\varphi\left(x_{1}\right), \ldots, \varphi\left(x_{n}\right)\right)$. For a bounded vector field $v$ of class $\mathcal{C}^{1}$ on $\mathbb{R}^{d}$, the infinitesimal action of $v$ at $q$ is given by $\xi_{q}(v)=\left(v\left(x_{1}\right), \ldots, v\left(x_{n}\right)\right)$. 
Most deformations can be "parametrized" as flows of time-dependent vector fields

$$
v=(v(t))_{t \in[0,1]}
$$

Moreover, if $\varphi$ is the flow associated to $v$, we get

$$
\dot{q}(t)=\frac{d}{d t}\left(\varphi(t) \circ q_{0}\right)=v(t) \circ\left(\varphi(t) \cdot q_{0}\right)=v(t) \circ q(t) .
$$

Therefore, we get the following control system on $M$

$$
q(0)=q_{0}, \quad \dot{q}(t)=v(t) \circ q(t), \quad \text { a.e. } t \in[0,1] .
$$

Usually, one takes $v$ to be at least in $L^{1}\left([0,1], \mathcal{C}_{b}^{1}\left([0,1], \mathbb{R}^{d}\right)\right)$, i.e. time-dependent bounded vector fields of class $\mathcal{C}^{1}$, integrable with respect to time, which ensures the existence of a flow.

The energy $E$ of a deformation can then be defined directly on the control. For this, we restrict ourselves to vector fields belonging to some Hilbert space $V$ of bounded vector fields of class $\mathcal{C}^{1}$, such as a Sobolev space $H^{k}\left(\mathbb{R}^{d}, \mathbb{R}^{d}\right)$ with $k$ big enough. The total energy of a time-dependent vector field $v$ is then defined by

$$
E(v)=\int_{0}^{1}\|v(t)\|^{2} d t
$$

Remark 1. It is possible to take more general energy, though one usually focuses on positive definite bilinear forms.

Mixed constraints can then be added to this system, defined by some $C: M \times V \rightarrow Y, Y$ a Banach space. We will only consider constraints linear with respect to the control (i.e. $v)$. We will denote $C(q, v)=C_{q} v$ to emphasize this last point.

We finally get our optimal control formulation of the shape deformation problem.

Problem 1. Let $q_{0} \in M$, and let $C: M \times V \rightarrow Y$ be a mapping such that $v \mapsto C(q, v)=C_{q} v$ is linear for every $q \in M$. Let $g: M \rightarrow \mathbb{R}$ be a function. We consider the problem of minimizing the functional

$$
J_{1}(v)=\frac{1}{2} \int_{0}^{1}\|v(t)\|_{V}^{2} d t+g(q(1))
$$

over all time-dependent vector fields $v(\cdot) \in L^{2}(0,1 ; V)$ such that $C_{q(t)} v(t)=0$ for every $t \in[0,1]$, where $q(\cdot):[0,1] \rightarrow M$ is the curve defined by $q(0)=q_{0}$ and $\dot{q}(t)=\xi_{q(t)} v(t)$ for almost every $t \in[0,1]$.

Remark 2. The problem could be posed for more general (i.e. non-linear) constraints $C(q, v)=0$. We focused on linear constraints because such constraints often have a clearer geometric meaning.

\section{Existence AND PRoperties of optimal CONTROLS}

In this section, we give sufficient conditions for the existence of a solution to Problem 1, and first-order necessary conditions for optimality, on the basis of the well-known Pontryagin Maximum Principle (PMP, see $[7,13])$ in optimal control theory. The extension to our infinite dimensional framework is however nontrivial. This allows us to derive the constrained geodesic equations for shape spaces.

Throughout the section, we adopt the framework and the notations defined in Section 1. Let $M$ be the shape space of a compact smooth Riemannian manifold $S$. Let $V$ be a Hilbert space of bounded vector fields of class $\mathcal{C}^{1}$ on $\mathbb{R}^{d}$, and $Y$ be a Banach space. Let $C: M \times V \rightarrow Y$ be a mapping such that $v \mapsto C(q, v)=C_{q} v$ is linear for every $q \in M$. Let $g: M \rightarrow \mathbb{R}$ be a function. 
Existence of an optimal control. Our first theorem establishes the existence of solutions to Problem 1 under minimal assumptions.

Theorem 1. If $q \mapsto C_{q}$ and $g$ are continuous, if $g$ is bounded from below, and if $V$ has a continuous inclusion in the space of $\mathcal{C}^{1}$ bounded vector fields with bounded derivatives, then Problem 1 has at least one solution.

This is a consequence of Ascoli's Theorem, combined with the usual proof for the existence of optimal control in finite dimensions (see [14]).

First-order optimality conditions. In this section, we state first-order necessary conditions for optimality in Problem 1 by extending the Pontryagin Maximum Principle to our setting.

For this, we need to define the hamiltonian $H: M \times M^{*} \times V \times Y^{*} \rightarrow \mathbb{R}$ of the problem by

$$
H(q, p, v, \lambda)=\left\langle p, \xi_{q} v\right\rangle_{M^{*}, M}-\frac{1}{2}\|v\|_{V}^{2}-\left\langle\lambda, C_{q} v\right\rangle_{Y^{*}, Y}
$$

Theorem 2. Assume that $g$ and $C$ are of class $\mathcal{C}^{1}$, and that $V$ has a continuous inclusion in the space of $\mathcal{C}^{1}$ bounded vector fields with bounded derivatives.

If $v \mapsto C_{q} v$ is surjective for all $q \in M$, then for any optimal control $v \in L^{2}(0,1 ; V)$, there exist $p \in$ $H^{1}\left(0,1 ; M^{*}\right)$ and $\lambda \in L^{2}\left(0,1 ; Y^{*}\right)$ such that $p(1)+d g_{q(1)}=0$ and

$$
\begin{aligned}
\dot{q}(t) & = & \partial_{p} H(q(t), p(t), v(t), \lambda(t)), \\
\dot{p}(t) & = & -\partial_{q} H(q(t), p(t), v(t), \lambda(t)), \\
0 & = & \partial_{v} H(q(t), p(t), v(t), \lambda(t)),
\end{aligned}
$$

for almost every $t \in[0,1]$.

The proof follows from the application of the infinite dimensional constrained extremum theorem combined with a careful analysis of the Lagrange multipliers.

Remark 3. The theorem should hold true for non-linear equality constraints $C(q, v)=0$, with the assumption that $\partial_{v} C_{q, v}$ is surjective for every $(q, v) \in M \times V$, but one must then consider controls in $L^{\infty}(0,1 ; V)$ instead of $L^{2}$.

Remark 4. Note that the surjectivity assumption is a strong one in infinite dimension. It is usually not satisfied in the case of shape spaces when $Y$ is infinite dimensional.

Remark 5. This is a "weak" maximum principle: we derive the condition $\partial_{v} H=0$ along any extremal, instead of the usual (stronger) maximization condition. However, in the case of shape spaces, $v \mapsto H(q, p, v, \lambda)$ is strictly concave and hence both conditions are equivalent.

Remark 6. Taking the energy to be the $L^{2}$-norm on vector fields of $\mathbb{R}^{d}$ instead of the norm on $V$, letting $C_{q} v=\operatorname{div} v$, and applying the theorem gives the usual incompressible Euler equations of fluid mechanics (see [2]).

The Geodesic Equations on a Shape Space. A careful analysis of the equations of the PMP allows us to express $v$ and $\lambda$ as functions of $q$ and $p$, with

$$
v_{q, p}=K_{V}\left(\xi_{q}^{*} p-C_{q}^{*} \lambda\right)
$$

and

$$
\lambda_{q, p}=\lambda_{q, p}=\left(C_{q} K_{V} C_{q}^{*}\right)^{-1} C_{q} K_{V} \xi_{q}^{*} p .
$$

Here, $\xi_{q}^{*}: M^{*} \rightarrow V^{*}$ is the pull-back induced by $\xi_{q}$ and $K_{V}$ is the inverse of the isometry $V \rightarrow V^{*}$ given by $v \mapsto\langle v, \cdot\rangle_{V}$. Also note that $C_{q}$ being surjective implies that $C_{q} K_{V} C_{q}^{*}$ is indeed invertible. 
This allows us to define the reduced Hamiltonian $h: M \times M^{*} \rightarrow \mathbb{R}$ as

$$
h(q, p)=H\left(q, p, v_{q, p}, \lambda_{q, p}\right),
$$

and prove that optimal trajectories satisfy the usual Hamiltonian equations [3].

Proposition 1. (Geodesic Equations in Shape Spaces) If the assumptions of Theorem 2 are satisfied, then for any trajectory $q:[0,1] \rightarrow M$ of a solution $v$ of Problem 1 , there is a map $p \in H^{1}\left(0,1 ; M^{*}\right)$ such that $p(1)+d g_{q(1)}=0$ and for a.e. $t \in[0,1]$ and $(q, p)$ satisfies the equations

$$
\left\{\begin{array}{l}
\dot{q}(t)=\partial_{p} h(q(t), p(t)), \\
\dot{p}(t)=-\partial_{q} h(q(t), p(t)) .
\end{array}\right.
$$

Such a couple $(q, p)$ is called a geodesic. Moreover, $t \mapsto \frac{1}{2}\|v(t)\|^{2}$ is constant. In particular, the total cost $J_{1}(v)$ of the trajectory is given by

$$
J_{1}(v)=\frac{1}{2}\|v(0)\|_{V}^{2}+g(q(1)) .
$$

This gives a minimization algorithm, called minimization by shooting. Indeed, assume that $C$ and all vector fields in $V$ are of class $\mathcal{C}^{2}$. Then an initial momentum $p_{0} \in M^{*}$ gives a unique geodesic $(q, p)$ from the initial state $q_{0}$. Now, if $q$ is an optimal trajectory if and only if it minimizes the reduced cost

$$
J_{2}\left(p_{0}\right)=\frac{1}{2}\left\|v_{q_{0}, p_{0}}\right\|_{V}^{2}+g(q(1)) .
$$

This can be done using a gradient descent for a particular inner product.

\section{Examples of Matching}

Constant Total Volume. For our first example, we consider a very simple constraint. Consider $S=S^{1}$ the unit circle in $\mathbb{R}^{2}$, and let $M=\mathcal{C}^{1}\left(S, \mathbb{R}^{d}\right)$ be our shape space. Note that we took a smaller shape space here, but our results still work, although with slightly stronger hypotheses (mainly, $V$ must have bounded derivatives of order 2 for theorems 1 and 2, and bounded derivatives of order 3 for existence and unicity of solutions to the geodesic equations).

Assume the initial state $q_{0}(s)=(x(s), y(s))$ is a simple closed curve of class $\mathcal{C}^{1}$. This property is conserved through deformation by diffeomorphism.

Such a curve is the boundary of an open subset $U(q)$ with total area given by $\operatorname{Vol}(U(q))=\int_{S} x(s) d y(s)$. Now, define a target $q_{1}$ such that $\operatorname{Vol}\left(U\left(q_{0}\right)\right)=\operatorname{Vol}\left(U\left(q_{1}\right)\right)$. We take the constraints $\operatorname{Vol}(U(q(t)))=\operatorname{Vol}\left(U\left(q_{0}\right)\right)$ for every $t \in[0,1]$.

We will consider $V$, a reproducing kernel Hilbert space of smooth vector fields given by the Gaussian kernel with positive scale $\sigma$ (see [17] for definitions).

For numerical applications, we will take $d=2$, so $S=S^{1}$ and $M$ is a space of curves, which will be discretized as landmarks $q=\left(x_{1}, \ldots, x_{n}\right) \in L m k_{2}(n)$. In this case, the volume of a curve is approximately equal to the volume of the polygon $P(q)$ with vertices $\left(x_{i}\right)$, given by

$$
\operatorname{Vol}(P(q))=\frac{1}{2}\left(x_{1} y_{2}-y_{2} x_{1}+\cdots+x_{n} y_{1}-y_{n} x_{1}\right) .
$$

Then, the shooting algorithm can be applied, giving the diffeomorphism shown in Figure 1.

Usually, a minimizing flow matching a circle on a translated circle will shrink it along the way. However, since we required the volume to be constant, the circle will look more like it is translated toward the target, though the diffeomorphism itself does not look like a tranlastion. Both trajectories are presented in Figure 2. 

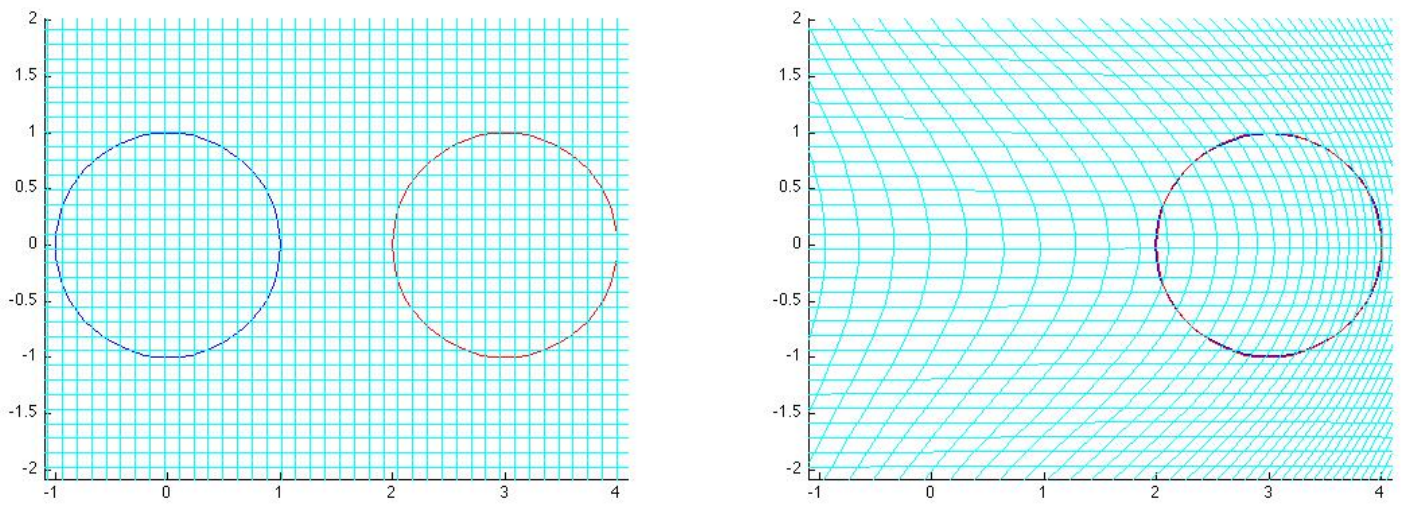

Figure 1. Constant volume experiment, left: initial (blue) and target (red), matching (right).
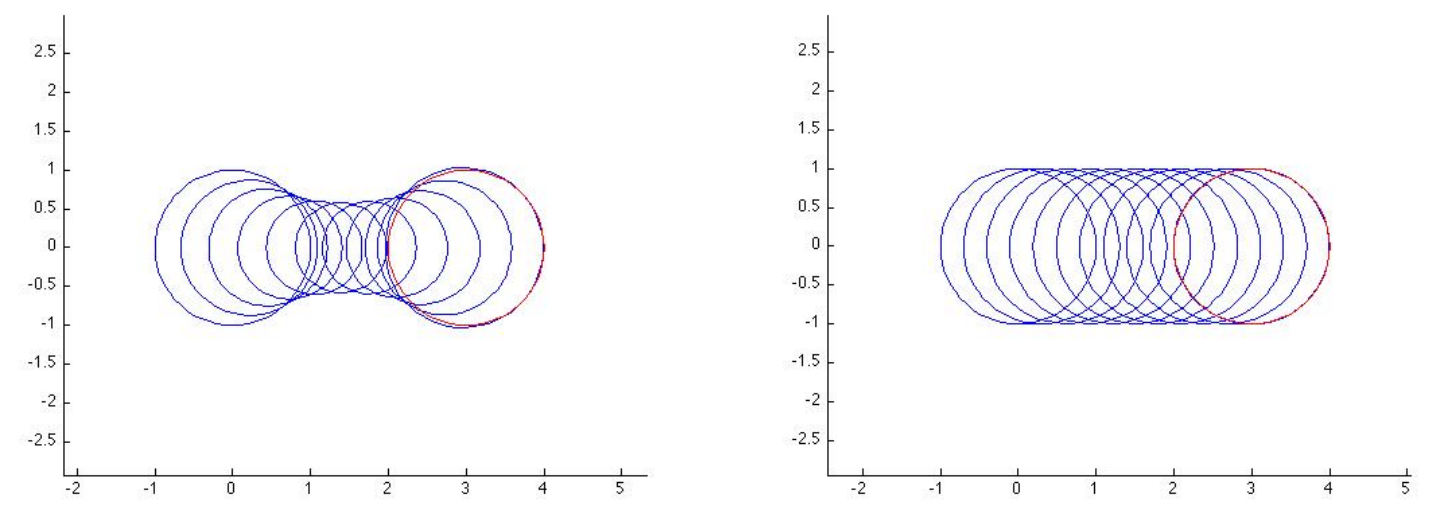

Figure 2. Matching trajectories without constraints (left) and with constant volume (right).

Multishape Matching. We now consider the multishape problem. In this situation, we consider shape spaces $M_{1}, M_{2}$, with $M_{1}=M_{2}=\mathcal{C}^{0}\left(S^{1}, \mathbb{R}^{2}\right)$ and a background space $M_{3}=M_{1} \times M_{2}$. Elements of $M_{3}$ are couples $q_{3}=\left(q_{3}^{1}, q_{3}^{2}\right)$, with $q_{3}^{j} \in M_{j}, j=1,2$.

We take an initial state $q_{0}=\left(q_{1}, q_{2}, q_{3}\right)$ satisfying $q_{3}^{1}=q_{1}$, and $q_{3}^{2}=q_{2}$.

To each $j \in\{1,2,3\}$ is associated a Hilbert space $V_{j}$ of vector fields, with total control space the Hilbert space $V_{1} \times V_{2} \times V_{3}$.

We will consider two types of compatibility constraints between homologous curves $q_{j}$ and $q_{3}^{j}$, namely

(1) Identity (or stitched) constraint: $q_{j}=q_{3}^{j}$

(2) Identity up to reparametrization (or sliding) constraint: $q_{3}^{j}=q_{j} \circ f$ for some (time-dependent) diffeomorphism of $S^{1}$.

Note that, since the curves have the same initial condition, the last constraint is equivalent to assuming that $\dot{q}_{3}^{j}-\dot{q}_{j} \circ f$ is tangent to $q_{3}^{j}$, i.e., taking controls $\left(v_{1}, v_{2}, v_{3}\right)$,

$$
\left(v_{3}\left(t, q_{3}^{j}\right)-v_{j}\left(t, q_{3}\right)^{j}\right) \cdot \nu_{3}^{j}=0
$$


for all $t$, where $\nu_{3}^{j}$ is the normal vector to $q_{3}^{j}$.

For the numerical computations, the curves are discretized into polygonal lines, and the discretization of control equations and objective functions are done by reduction to landmarks space. The discretization of the constraint in the identity case is straightforward. For each $j=1,2$ and each line segment $\ell=\left[z_{\ell}^{-}, z_{\ell}^{+}\right]$in $q_{3, j}$ (represented as a polygonal line), we simply use the constraint

$$
\nu_{\ell} \cdot\left(v_{j}(\ell)-v_{3}(\ell)\right)=0
$$

where $\nu_{\ell}$ is the unit normal to $\ell$ (unit vector perpendicular of $z_{\ell}^{+}-z_{\ell}^{-}$) and

$$
v_{j}(\ell)=\frac{1}{2}\left(v_{j}\left(z_{\ell}^{-}\right)+v_{j}\left(z_{\ell}^{+}\right)\right) .
$$

Note that the vertices $z_{\ell}^{-}$and $z_{\ell}^{+}$are already part of the state variables that are obtained after discretizing the background boundaries $q_{3}$.

Given these choices, we can apply a minimization algorithm. In Figure 3, we provide an example comparing the two constraints. The desired transformation, as depicted in Figure 3, moves a curve with elliptical shape upward, and a flower-shaped curve downward, each curve being subject to a small deformation. The solutions obtained using the stitched and sliding constraints are provided in Figure 4, in which we have also drawn a deformed grid, representing the diffeomorphisms induced by the vector fields $v_{1}, v_{2}$ and $v_{3}$ in their relevant regions.

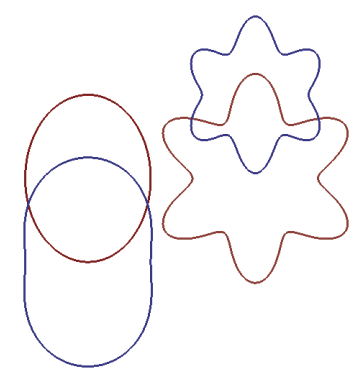

Figure 3. Multishape Experiment: Initial (blue) and target (red) sets of curves.

\section{REFERENCES}

[1] S. Arguillère, E. Trélat, A. Trouvé, L. Younès, Shape deformation analysis from the optimal control viewpoints, preprint (2013).

[2] V. Arnold, Sur la géométrie différentielle des groupes de Lie de dimension infinie et ses applications à l'hydrodynamique des fluides parfaits, Ann. Inst. Fourier 16 (1966), fasc. 1, 319-361.

[3] V. Arnold, Mathematical methods of classical mechanics, Graduate Texts in Mathematics 60, Springer-Verlag, New York, 1989.

[4] B. Avants, J.C. Gee, Geodesic estimation for large deformation anatomical shape averaging and interpolation, Neuroimage 23 (2004), S139-S150.

[5] M.F. Beg, M.I. Miller, A. Trouvé, L. Younès, Computing large deformation metric mappings via geodesic flows of diffeomorphisms, Int. J. Comput. Vis. 61 (2005), no. 2, 139-157.

[6] P. Dupuis, U. Grenander, M.I. Miller, Variational problems on flows of diffeomorphisms for image matching, Quart. Appl. Math. 56 (1998), no. 3, 587-600. 

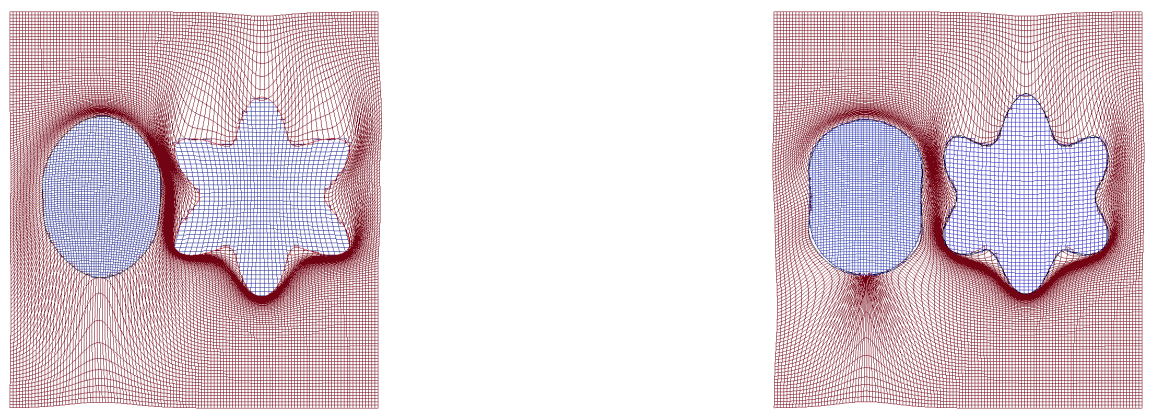

Figure 4. Multishape Experiment, stitched (left) and sliding (right) constraints.

[7] R.F. Hartl, S.P. Sethi, R.G. Vickson, A survey of the maximum principles for optimal control problems with state constraints, SIAM Rev. 37 (1995), no. 2, 181-218.

[8] D.D. Holm, J. Marsden, T.S. Ratiu, Euler-Poincaré models of ideal fluids with nonlinear dispersion, Phys. Rev. Lett. 80 (1998), no. 19, 4173-4176.

[9] S.C. Joshi, M.I. Miller, Landmark matching via large deformation diffeomorphisms, IEEE Transcript Image Processing 9 (2000), no. 8, 1357-1370.

[10] J.E. Marsden, T.S. Ratiu, Introduction to mechanics and symmetry, Texts in Applied Mathematics 17, second edition, Springer-Verlag, New York, 1999.

[11] M.I. Miller, A. Trouvé, L. Younès, On the metrics and Euler-Lagrange equations of computational anatomy, Annu. Rev. Biomed. Eng. 4 (2002), 375-405.

[12] M.I. Miller, A. Trouvé, L. Younès, Geodesic shooting for computational anatomy, J. Math. Imaging Vision 24 (2006), no. 2, 209-228.

[13] L.S. Pontryagin, V.G. Boltyanskii, R.V. Gamkrelidze, E.F. Mishchenko, The mathematical theory of optimal processes, A Pergamon Press Book, The Macmillan Co., New York, 1964.

[14] E. Trélat, Contrôle optimal (French) [Optimal control], Théorie $\&$ applications [Theory and applications], Math. Concrètes [Concrete Mathematics], Vuibert, Paris, 2005.

[15] A. Trouvé, Action de groupe de dimension infinie et reconnaissance de formes, C. R. Acad. Sci. Paris Sér. I Math. 321 (1995), no. 8, 1031-1034.

[16] A. Trouvé, Diffeomorphism groups and pattern matching in image analysis, International Journal of Computational Vision 37 (2005), no. 1, 17 pages.

[17] A. Trouvé, L. Younès, Shape spaces, in: Handbook of Mathematical Methods in Imaging, O. Scherzer ed., Springer New York, 2011, 1309-1362.

[18] L. Younès, Shapes and diffeomorphisms, Applied Mathematical Sciences 171, Springer-Verlag, Berlin, 2010.

[19] L. Younès, Constrained diffeomorphic shape evolution, Found. Comput. Math. 12 (2012), no. 3, $295-325$. 